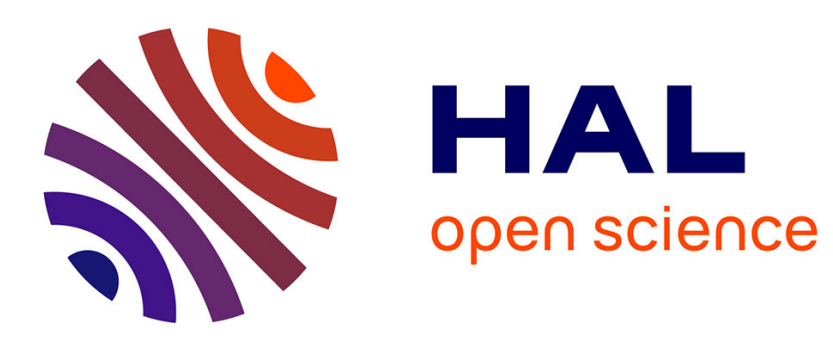

\title{
Electrophoretic mobility of silica particles in a mixture of toluene and ethanol at different particle concentrations
}

\author{
M. Medrano, A.T. Perez, Laurent Lobry, Francois Peters
}

\section{- To cite this version:}

M. Medrano, A.T. Perez, Laurent Lobry, Francois Peters. Electrophoretic mobility of silica particles in a mixture of toluene and ethanol at different particle concentrations. Langmuir, 2009, 25 (20), pp.12034-12039. 10.1021/la900686a . hal-00442624

\section{HAL Id: hal-00442624 \\ https://hal.science/hal-00442624}

Submitted on 21 Dec 2009

HAL is a multi-disciplinary open access archive for the deposit and dissemination of scientific research documents, whether they are published or not. The documents may come from teaching and research institutions in France or abroad, or from public or private research centers.
L'archive ouverte pluridisciplinaire HAL, est destinée au dépôt et à la diffusion de documents scientifiques de niveau recherche, publiés ou non, émanant des établissements d'enseignement et de recherche français ou étrangers, des laboratoires publics ou privés. 


\title{
Electrophoretic mobility of silica particles in a
}

\author{
mixture of
}

\section{toluene and ethanol at different particle}

\section{concentrations}

\author{
M. Medrano, ${ }^{\dagger}$ A. T. Pérez, ${ }^{* \dagger}{ }^{\dagger}$ L. Lobry, ${ }^{\ddagger}$ and F. Peters ${ }^{\ddagger}$ \\ Departamento de Electrónica y Electromagnetismo, Universidad de Sevilla (Spain), and \\ Laboratoire de Physique de la Matière Condensé, CNRS-Université de Nice (France) \\ E-mail: alberto@us.es
}

\begin{abstract}
In this paper we present measurements of the electrophoretic mobility of colloidal particles by using heterodyne detection of light scattering. The measurements have been done up to concentrations of $5.4 \%$ of silica nanoparticles, with a diameter of the order of $80 \mathrm{~nm}$, in a mixture of $70 \%$ toluene and $30 \%$ ethanol. In order to make possible the measurements at these concentrations the liquid mixture is chosen as to match the index of refraction of the particles, thus resulting in a transparent suspension.
\end{abstract}

\footnotetext{
${ }^{\dagger}$ Universidad de Sevilla

†Université de Nice
} 


\section{Introduction}

Particles of various sorts often acquire charge when are immersed in low conducting liquids. The electric repulsion between the particles contributes to stabilize the suspension. Although this stabilization is in general weaker than for aqueous media, it is of importance in some industrial areas, such as printing and xerography, and from a fundamental point of view.

The charge on the particle surface induces the accumulation of ions of opposite sign around the particle. This gives rise to a structure known as the double layer. When an electric field is applied to a suspension of charged particles, a force appears on both parts of the double layer. This force moves the particles with respect to the liquid with a velocity proportional to the applied field. The coefficient of proportionality is referred to as the electrophoretic mobility. This phenomenon was observed for the first time by Reuss in 1809. Smoluchowski developed the first theory of electrophoresis for one insulated particle when the zeta potential is small and the particle radius is much larger than the Debye length, $\kappa a \gg 1$ ( $a$ is the particle radius and $\kappa^{-1}$ is the Debye length). His well known solution for the electrophoretic mobility is

$$
\mu_{E}=\frac{\varepsilon_{r} \varepsilon_{0} \zeta}{\eta}
$$

where $\mu_{E}$ is the electrophoretic mobility, $\varepsilon_{r}$ is the relative permittivity of the liquid, $\varepsilon_{0}$ is the dielectric permittivity of the vacuum, $\zeta$ is the zeta potential and $\eta$ is the viscosity of the liquid. On the other hand, Hückel obtained the expression of the mobility for particles with a thick double layer $(\kappa a \ll 1)$

$$
\mu_{E}=\frac{2}{3} \frac{\varepsilon_{r} \varepsilon_{0} \zeta}{\eta}
$$

Later, Henry joined both electrophoresis relations within an analytical expression that is valid for a single sphere with small zeta potentials and arbitrary double layer width. He included the function $f(\kappa a)$ in the Smoluchowski expression $\mu_{\text {Henry }}=\mu_{\text {Smol }} f(\kappa a)$. Function $f(\kappa a)$ is known as the Henry function, this function is 1 when $\kappa a \rightarrow \infty$ (Smoluchowski approximation) and it is 
$2 / 3$ when $\kappa a \rightarrow 0$ (Hückel approximation). For thick double layers $(\kappa a<5)$, the Henry function is 1

$$
\begin{aligned}
f(\kappa a)=\frac{2}{3} & +\frac{(\kappa a)^{2}}{24}-\frac{5(\kappa a)^{3}}{72}-\frac{(\kappa a)^{4}}{144}+\frac{(\kappa a)^{5}}{144} \\
& +\left[\frac{(\kappa a)^{4}}{12}-\frac{(\kappa a)^{6}}{144}\right] e^{\kappa a} \int_{\infty}^{\kappa a} \frac{e^{-x}}{x} \mathrm{~d} x \ldots
\end{aligned}
$$

Both Smoluchowski and Henry solutions did not include hydrodynamic interactions between neighboring particles.

For a finite volume fraction, even without taking into account hydrodynamic interactions or double layer overlap, the electrophoretic mobility depends on the volume fraction for the following reason: when the particle moves in one direction, the same volume of liquid has to move in the opposite direction. This back flow results in a dependence of the mobility on the particle concentration as ${ }^{5-8}$

$$
\mu_{E}(\varphi)=\mu_{E}(0)[1-\varphi]
$$

where $\varphi$ is the particle solid fraction.

Additionally, when the suspension is composed of insulating particles, they alter the distribution of the applied electric field. ${ }^{8}$ This fact contributes a $-\varphi / 2$ to the expression of $\mu_{E}$

$$
\mu_{E}(\varphi)=\mu_{E}(0)\left[1-\frac{3}{2} \varphi+O\left(\varphi^{2}\right)\right]
$$

\section{where $O\left(\varphi^{2}\right)$ denotes terms of order $\varphi^{2}$ and smaller.}

Reed and Morrison ${ }^{3}$ studied the hydrodynamic interactions for pairs of particles with a thin double layer as function of the interparticle distance. They showed that hydrodynamic and electric interactions cancel each other when the particles have the same zeta potential. In that case, equation (5) is expected to describe the electrophoretic mobility.

However, when the particles have a thick double layer, the interaction between them becomes more complex. As a consequence of the interactions, the electrophoretic mobility decreases as $\varphi$ 
increases more quickly than predicted by equation (5). In this case, the mobility is 4,9

$$
\mu_{E}(\varphi)=\mu_{E}(0)\left[1+S \varphi+O\left(\varphi^{2}\right)\right]
$$

where the coefficient $S$ depends on the parameter $\kappa a$. When $\kappa a$ is smaller than 20 , this coefficient decreases quickly. The case of $\kappa a$ as low as 1 has been numerically addressed in Shugai's work. ${ }^{4}$ For $\kappa a=1, S$ is much less than $-3 / 2$.

For the case of the aqueous media there are some experimental electrophoretic studies, where the effect of the particle concentration on the mobility is measured. ${ }^{6,10}$ In our work, we focus on non aqueous systems.

For concentrate suspensions the use of the electrokinetic sonic amplitude (ESA) effect is a suitable technique of measurement. ${ }^{11}$ However, ESA works in the MHz range and the results rely on some theoretical assumptions concerning the generation of the sonic wave. Optical techniques are still of application for concentrate suspensions if the liquid is chose as to match the index of refraction of the particles, rendering the suspension transparent.

We study suspensions of silica in a mixture of ethanol and toluene with a technique of photon correlation spectroscopy (PCS). In spite of the fact that there are some commercial apparatuses available, we have used our own system for two reasons. First, we can apply higher electric voltages. Second, we have a better control of the data analysis.

We will first recall the theoretical background concerning the PCS technique. Then we present the materials and the experimental set-up, followed by the experimental results. In the last section, we discuss them in the light of existing theoretical model and numerical computing based on hydrodynamic interactions and double layer overlap.

\section{Theoretical Background}

The photon correlation spectroscopy (PCS) is a useful method to characterize colloidal suspensions, micellar systems or biological materials. In PCS, a light beam is directed towards the 
sample and the scattered light is detected with an appropriate device, usually a photomultiplier.

There are two different PCS methods: homodyne and heterodyne. In the homodyne method only the scattered light impinges on the photocathode. In the heterodyne method, a portion of the unscattered light is mixed with the scattered light on the photomultiplier cathode.

For a set of uncorrelated particles, the autocorrelation function of the light intensity measured by the photomultiplier in heterodyne detection is (see the Appendix for details):

$$
C_{2}(\tau)=1+\alpha_{2} e^{-\Gamma \tau} \cos \left(\omega_{D} \tau\right)
$$

where $\alpha_{2}$ is a constant that depends on some experimental parameters, the constant $\Gamma$ is the product $D q^{2}$, and the Doppler frequency $\omega_{D}$ is defined as

$$
\omega_{D}=\vec{q} \cdot \vec{v}=v q \cos \alpha
$$

where $\alpha$ is the angle between the scattering vector $\vec{q}$ and the particle velocity $\vec{v}$.

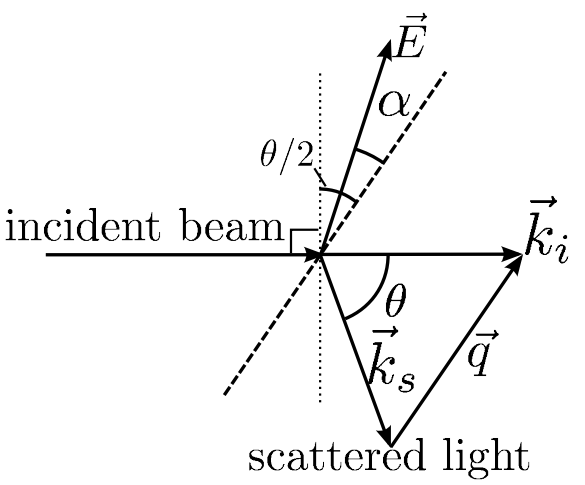

Figure 1: Geometry for an electrophoretic light scattering experiment.

When particle move by electrophoresis their velocity is $\vec{v}=\mu_{E} \vec{E}$. If the applied electric field is perpendicular to the incoming beam, the angle $\alpha$ is $\theta / 2$ (see Figure 1). Then the Doppler 
frequency is

$$
\omega_{D}=\frac{2 \pi n}{\lambda_{0}} \mu_{E} E \sin \theta
$$

where $E$ is the module of the electric field and $\theta$ is the angle between the incoming and scattered beams. Here, we have supposed that $\left|\vec{k}_{i}\right| \approx\left|\vec{k}_{s}\right|$, then the scattering vector module is $|\vec{q}|=$ $\left(4 \pi n / \lambda_{0}\right) \sin (\theta / 2)$, where $\lambda_{0}$ is the wave length in the vacuum of the incoming beam, and $n$ is the refraction index of the scattering medium.

In order to obtain $\Gamma$ and $\omega_{D}$, we study the real part of the Fourier transform $G_{2}(\omega)$ of the correlation function $C_{2}(\tau)-1$ :

$$
\begin{aligned}
\operatorname{Re}\left[G_{2}(\omega)\right]= & \alpha_{2}\left(\frac{\Gamma}{\Gamma^{2}+\left(\omega-\omega_{D}\right)^{2}}\right. \\
& \left.+\frac{\Gamma}{\Gamma^{2}+\left(\omega+\omega_{D}\right)^{2}}\right)
\end{aligned}
$$

This function is a Lorentzian with the peak placed on $\omega_{D}$.

If we define the width at mid-height $\omega_{1 / 2}^{(2)}$ as the frequency at which the value of $\operatorname{Re}\left[G_{2}(\omega)\right]$ is half the maximum value, equation (10) implies that $\omega_{1 / 2}^{(2)}=\Gamma$.

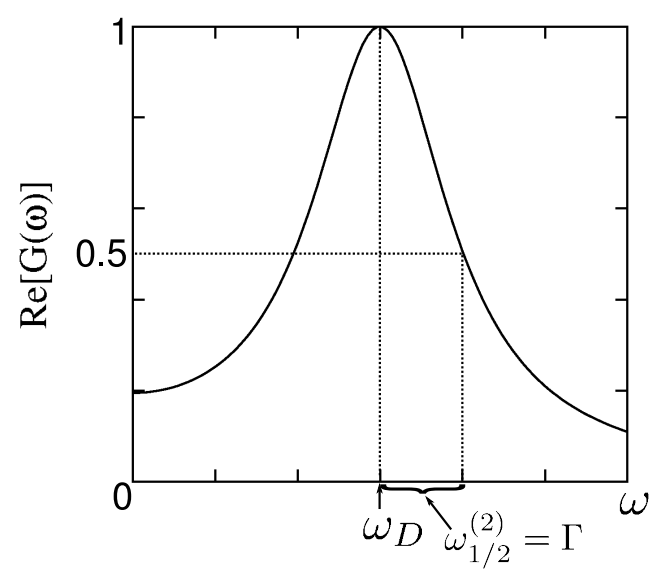

Figure 2: The normalized Fourier transform of the heterodyne correlation function, $C_{2}(t)-1$. It shows a peak at the Doppler frequency. 


\section{Experiment}

\section{Materials}

It is often assumed that optical methods are only useful for dilute suspensions, when the sample is transparent. However, it is possible to have a concentrated, yet transparent suspension. The requirement is that the refraction index of the liquid matches that of the particle. ${ }^{12}$

We have synthesized spherical and monodispersed silica nanoparticles by the Stöber method, ${ }^{13}$ which consist in hydrolyse tetraethyl-ortosilicate (TEOS) in ethanol, by using ammonia as catalyst. The final product is a suspension of silica particles in a solution of ethanol, water, and ammonia. This suspension is called alcosol. The proportion of reactants that we used was $0.5 \mathrm{M}$ of TEOS (supplied by Fluka), 0.1 $\mathrm{M}$ of distilled water, and $0.2 \mathrm{M}$ of $\mathrm{NH}_{3}(\mathbf{3 0} \%)$ in absolute ethanol. Ammonia and ethanol were supplied by Panreac.

For preparing the alcosol, we made two different solutions. The first contained $539 \mathrm{ml}$ of ethanol, $22 \mathrm{ml}$ of $\mathrm{NH}_{3}$, and $31 \mathrm{ml}$ of water. The second had $461 \mathrm{ml}$ of ethanol and $130 \mathrm{ml}$ of TEOS. The second solution was added to the first one while this was being stirred with a magnetic stirrer. The final mixture was stirred for $3.5 \mathrm{~h}$. The reaction yields $0.31 \mathrm{~g}$ of silica from $10 \mathrm{ml}$ of alcosol. In a second step, the particle surface was modified with phenyl groups in order to improve the suspension stability and to increase the hydrophobity of the particle surface. ${ }^{14}$ Specifically, we added $2.75 \mathrm{ml}$ of phenyltriethoxysilane supplied by Fluka. Under stirring, we left the reaction for one day. The polymer forms a layer on the particle surface providing steric stabilization.

In order to increase the particle concentration, the suspension is centrifuged at $2000 \mathrm{rpm}$ and the supernatant is removed. After each centrifugation, the suspension was redispersed by applying sonication for several minutes. It is important to prevent the particles from forming a compact layer at the bottom of the centrifuge tube, because the redispersion would be almost impossible to achieve. The next step is to change the remanent liquid by absolute ethanol. To this end, we centrifuged the suspension and replaced the supernatant by ethanol. The solid fraction of silica in the absolute ethanol was measured by evaporating a known volume of suspension and by weighing 
the dried residue. With this procedure the particle concentration obtained was of $18 \%$ in volume, where we have taken a silica density of $2.0 \mathrm{~g} / \mathrm{cm}^{3} .15$

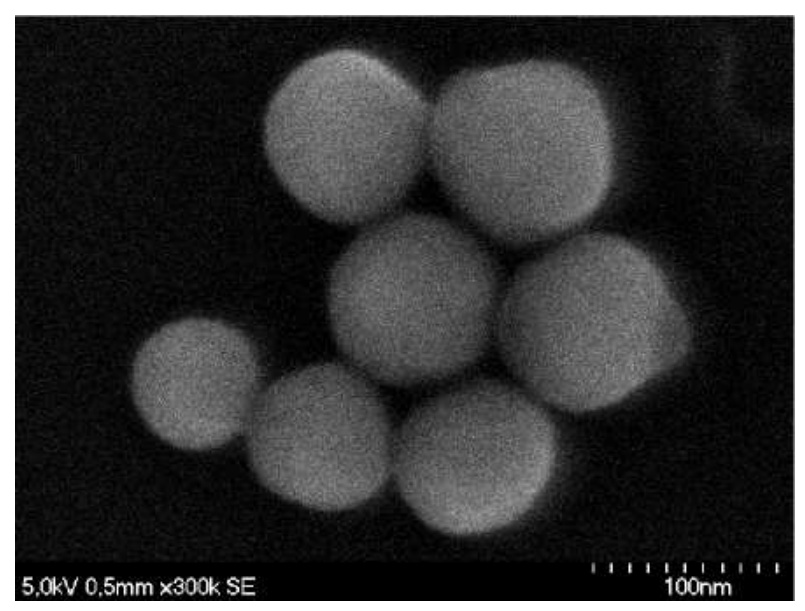

Figure 3: SEM micrograph of the particles.

The particle size was determined by analyzing a set of Scanning Electron Microscopy (SEM) photographs. Figure 3 shows one of this photographs. The diameter obtained was $(88 \pm 8) \mathrm{nm}$. The refractive index of the particles is 1.46 and its density is $2.0 \mathrm{~g} / \mathrm{cm}^{3}$.

The suspension of silica in ethanol is completely opaque. This is due to the difference between the particle and the liquid refractive indexes, 1.46 and 1.36 respectively. ${ }^{15}$ In order to match the refractive indexes, the suspending liquid was chosen as a mixture of toluene (70\%) and ethanol $(30 \%) .{ }^{16}$ For such a liquid, we did not observe multiple scattering, and the scattered intensity was sufficiently high for measurements to be performed. The conductivity of this mixture was measured to be $6.7 \pm 0.3 \mu \mathrm{S} / \mathrm{m}$ and the relative permittivity was $6.8 \pm 0.5$. Both parameters were measured with a device designed and built by the authors. ${ }^{17,18}$

\section{Experimental set-up}

Figure 4 shows a diagram of the experimental set-up. The main laser beam is divided into two beams by means of a beam splitter. The first beam comes to the measurement cell, and a lens focusses it at mid-distance between the cell electrodes. The second beam is led directly to the 


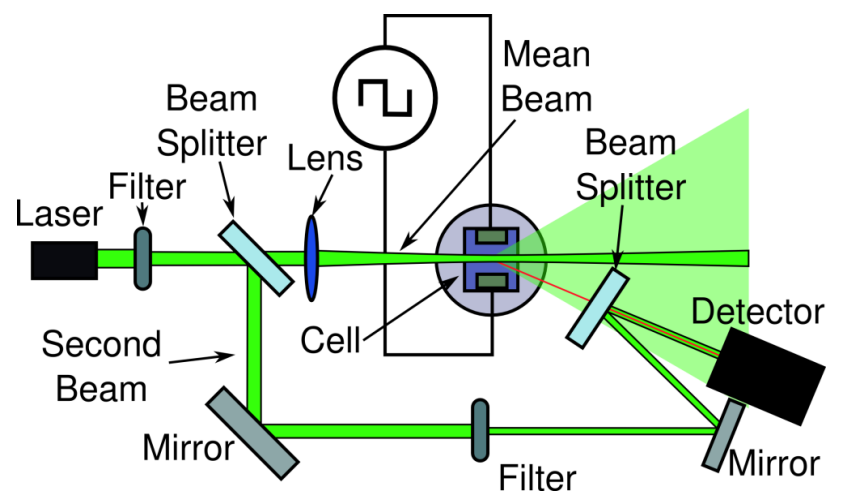

Figure 4: Diagram of the experimental set-up used for the heterodyne detection.

photomultiplier with the help of two mirrors. On the photomultiplier, the second beam and the light scattered by the sample are mixed together. The electric signal produced by the photomultiplier is sent to the correlator board inside the computer. The correlator counts the number of pulses and computes the autocorrelation function.

A set of diaphragms and lens are used to focus the scattered light onto the photomultiplier window and to choose the scattering volume of the cell.

The light intensity is regulated with two filters: one is in front of the laser and the other is placed on the secondary beam path. These filters allow to choose the intensity ratio between the scattered light and the secondary light beam. In heterodyne detection, the intensity of the scattered beam must be at least ten times lower than the secondary beam. Thus, the filters have to be changed for each sample, because the scattered light intensity varies with particle concentration.

The measurement cell is a dip cell suitable for organic liquids supplied by Malvern. It has a square section of $1 \mathrm{~cm}$ side and an electrode gap of $2 \mathrm{~mm}$. In order to minimize the refraction of the light, the cell is placed into the center of a cylindrical ethanol bath. The best would have been to use a bath with the same solution toluene-ethanol, but we came up against serious difficulties with the toluene vapor, because it dissolves some plastic pieces that supports the measurement cell. We control the angle of the scattered light with the help of goniometer, in our case we use an angle of $25^{\circ}$. For the calculations it is necessary to correct the scattering angle due to the refraction between the sample $(n=1.46)$ and the ethanol bath $(n=1.36)$. The corrected scattering angle is $23.18^{\circ}$. 
We applied for $50 \mathrm{~s}$ a symmetric square signal with a frequency of $1 \mathrm{~Hz}$. The temperature is measured and monitored for each measurement, always this has been between 18 and $20{ }^{\circ} \mathrm{C}$.

\section{Results and Discussion}

\section{Doppler frequency measurement}

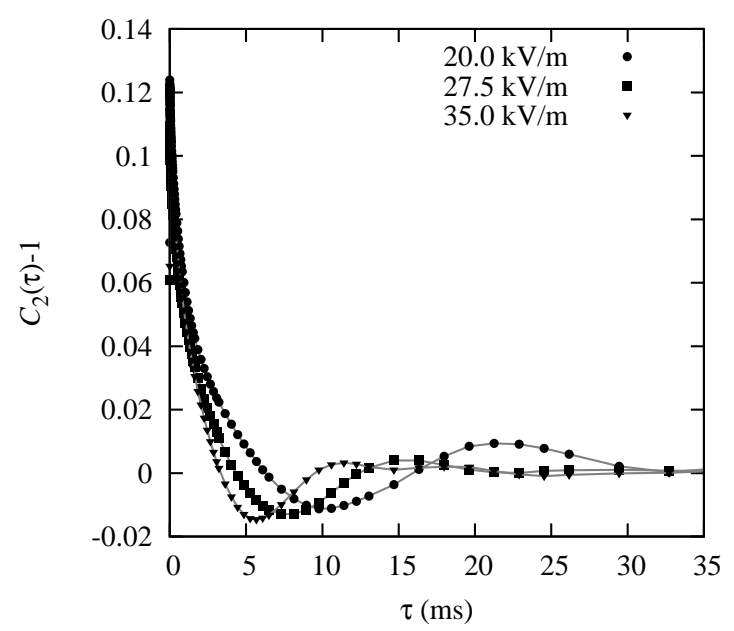

Figure 5: Example of some heterodyne correlation functions when the particles are undergoing electrophoresis. In this case the suspension has a concentration of particles of $4.5 \%$ in volume. The electrical signal is a symmetric square one with a frequency of $1 \mathrm{~Hz}$ and an amplitude of $20.0,27.5$ and $35.0 \mathrm{KV} / \mathrm{m}$.

Figure 5 shows several heterodyne correlation functions computed by the correlator, when the particles are undergoing electrophoresis. In order to obtain the Doppler frequency from the data set, we compute the Fourier transform of the heterodyne correlation function. According to equation (10), we identify the frequency of the maximum with the Doppler frequency. Figure 6 shows the spectrum of the correlation function for a voltage of $50 \mathrm{~V}$ between the electrodes $(25 \mathrm{KV} / \mathrm{m})$.

\section{Mobility measurement}

In order to determine the electrophoretic mobility, we measure the Doppler frequency for several electric fields. Figure 7 plots the Doppler frequencies versus the applied electric field for a suspen- 


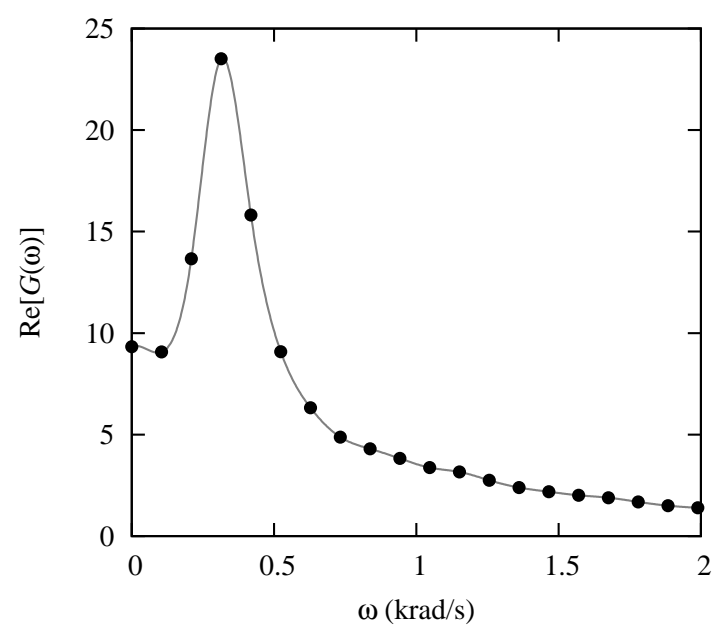

Figure 6: The function $G(\omega)$ is the Fourier transform of $C_{2}(\tau)-1$. This figure corresponds to the data obtained for an applied electric field of $25 \mathrm{KV} / \mathrm{m}$ to our suspension. The particle concentration is $4.5 \%$ in volume. It shows a peak at the Doppler frequency.

sion with a particle concentration $4.5 \%$ in volume. The frequency values have a linear relation with the applied voltage, as relation (9) predicts. The electrophoretic mobility is obtained from the slope of this straight line.

By using this method, we have obtained the electrophoretic mobility for several particle concentrations. Specifically, the particle concentrations was varied from $0.32 \%$ to $5.4 \%$ in volume.

The values of the mobility measurements with their error bars, are shown in Figure 8. For certain values of the electric field, we have also measured the mobility of the samples using a nano zetasizer of Malvern. The results are compatible with values obtained by PCS method. The zetasizer allows to determine the sign of the particle charge, which is negative.

Figure 8 shows how the electrophoretic mobility decreases with the particle concentration. Since we worked with semi-dilute suspensions $(\varphi<0.06)$, the values of the electrophoretic mobility can be fitted to relation (6) discarding the term $O\left(\varphi^{2}\right)$ :

$$
\begin{gathered}
\left|\mu_{E}(0)\right|=(2.7 \pm 0.2) 10^{-9} \mathrm{~m}^{2} / \mathrm{Vs} \\
S=-8 \pm 2
\end{gathered}
$$

This shows that $\mu_{E}$ decreases with particle concentration faster than predicted by relation (5), 


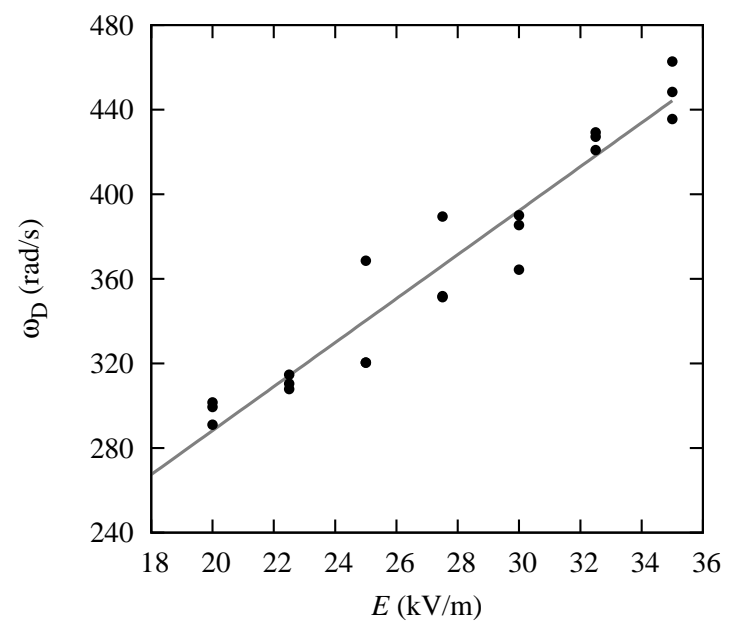

Figure 7: Doppler frequency versus applied electric field between the electrodes. The particle concentration is 4.5 $\%$ in volume. The straight line is the least square fit to the data. We have taken several measurements of $\omega_{D}$ for each value of the electric field.

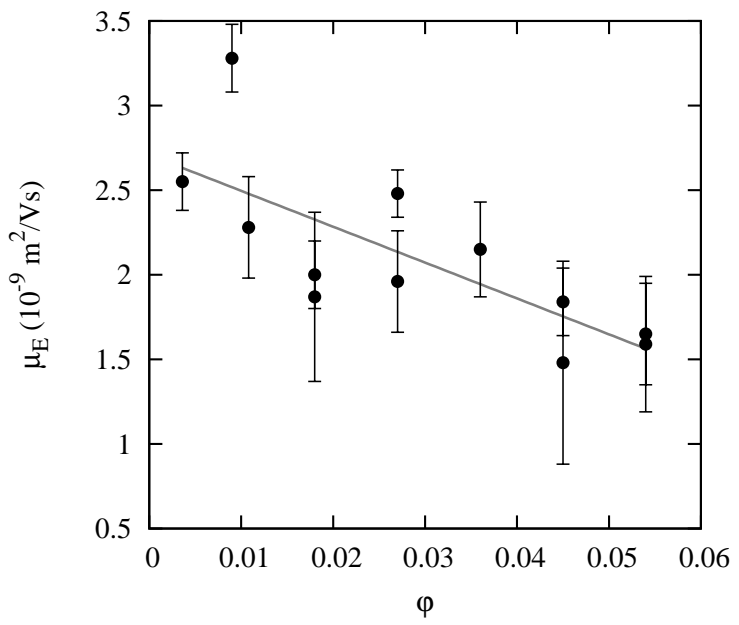

Figure 8: Electrophoretic mobility versus solid fraction. Each point in the plot corresponds to a different sample. 
which corresponds to $\kappa a \gg 1$.

\section{Mobility polydispersity}

When the applied electric field increases the Doppler frequency also increases. At first sight, and since the factor $e^{-\Gamma \tau}$ in equation (27) does not depend on the electric field, the heterodyne autocorrelation function should have more visible oscillations as the electric field increases. Therefore, for higher electric field, we expect that the spectrum peaks will lie farther from the origin and be better resolved. But Figure 5 shows that the number of visible oscillations in the correlation function does not increase with the electric field. In fact this figure shows that the amplitude of the correlation function decreases faster for larger fields. Moreover, what it is observed experimentally is a broadening of the peaks in the Fourier transform of the correlation function as the electric field increases (see Figure 9). Besides, a decrease in the value of the maximum value is found.

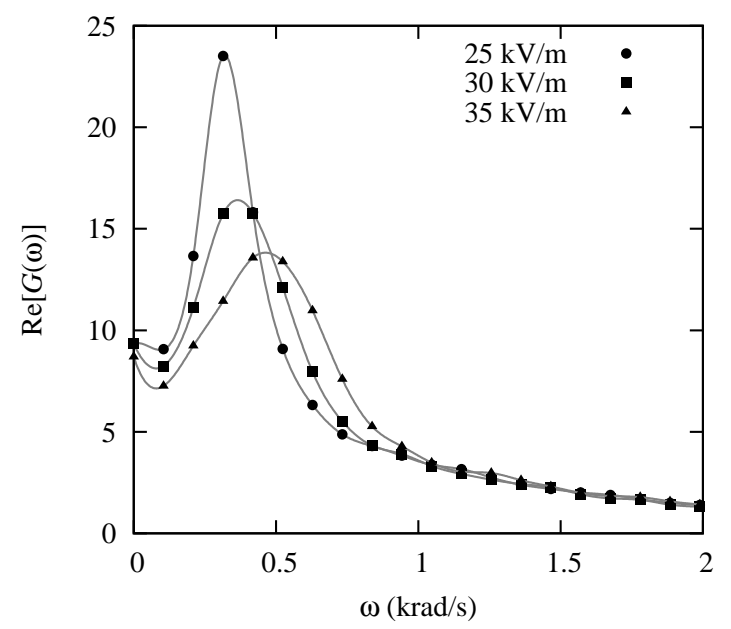

Figure 9: Fourier transform of the heterodyne correlation function for three values of the electric field. The Doppler frequency increases with the electric field, and the peaks become smaller and wider.

This effect was noticed in other works, ${ }^{10,20}$ where it is attributed to a diffusion coefficient dependence on the electric field. Effectively, accordingly to equation (10), the coefficient $\Gamma$ increases apparently with the applied voltages. Furthermore, the equation (10) implies that the broadening of the peaks is due to increase of $\Gamma$. However, this is not physically sound, since the diffusion 
coefficient is directly related to the particle size.

On the other hand, $\mathrm{Wu}^{21}$ suggests that a broadening of the peaks in heterodyne spectra is due to a mobility polydispersity. In fact, the expression (26) is valid only if the particles have all the same electrophoretic velocity. But, if there is a electrophoretic velocity distribution or, in other words, a Doppler frequency distribution, the effect of this polydispersity will affect the correlation functions.

To analyze the effect let us assume a Gaussian distribution of Doppler frequencies

$$
P\left(\omega_{D}\right)=\frac{1}{\sqrt{2 \pi} \sigma_{\omega}} e^{-\frac{\left(\omega_{D}-\omega_{D 0}\right)^{2}}{2 \sigma_{\omega}^{2}}}
$$

where $\omega_{D 0}$ is the average Doppler frequency and $\sigma_{\omega}$ is its dispersion. The new correlation function of the electric field is

$$
\begin{array}{r}
g(t)=\int_{-\infty}^{+\infty} \frac{1}{\sqrt{2 \pi} \sigma_{\omega}} e^{-\frac{\left(\omega_{D}-\omega_{D 0}\right)^{2}}{2 \sigma_{\omega}^{2}}} e^{\left(-\Gamma+i \omega_{D}\right) t} \mathrm{~d} \omega_{D} \\
=e^{-\Gamma t} e^{-\sigma_{\omega}^{2} t^{2}} e^{i \omega_{D 0} t}
\end{array}
$$

With this new expression of the correlation function of the light electric field and equation (23), the expression of the correlation function for heterodyne detection is

$$
C_{2}(t)=1+\alpha_{2} \operatorname{Re}[g(t)]=1+\alpha_{2} e^{-\Gamma t-\sigma_{\omega}^{2} t^{2}} \cos \left(\omega_{D 0} t\right)
$$

This expression shows that the relaxation time of the correlation functions depends on the Doppler frequency dispersion.

The Fourier transform of correlation function (13) has not an analytical expression. But we can calculate the limits when $\sigma_{\omega} \ll \Gamma$ and $\sigma_{\omega} \gg \Gamma$. For the first case, we can take $\sigma_{\omega} \rightarrow 0$, then the Fourier transform will be relation (10). In the second case, where the frequency dispersion 
dominates, we can take the limit $\Gamma \rightarrow 0$, then the Fourier transform of equation (13) is

$$
\begin{aligned}
\operatorname{Re}\left[G_{2}(\omega)\right]=\frac{1}{2} \frac{\sqrt{2 \pi}}{\sigma_{\omega}}[ & \exp \left(-\frac{\left(\omega-\omega_{D 0}\right)^{2}}{2 \sigma_{\omega}^{2}}\right)+ \\
& \left.\exp \left(-\frac{\left(\omega+\omega_{D 0}\right)^{2}}{2 \sigma_{\omega}^{2}}\right)\right] .
\end{aligned}
$$

For high electric fields, where we can neglect the diffusion term in the autocorrelation functions, the frequency $\omega_{1 / 2}^{(2)}$ is determined by the frequency dispersion

$$
\omega_{1 / 2}^{(2)}=\sigma_{\omega} \sqrt{2 \ln 2}
$$

The interesting point is that $\sigma_{\omega}$ depends on the electric field, since

$$
\begin{gathered}
\omega_{D}=\frac{2 \pi n}{\lambda} \sin (\theta) \mu_{E} E \\
\sigma_{\omega}=\frac{2 \pi n}{\lambda} \sin (\theta) \sigma_{\mu} E,
\end{gathered}
$$

where $\mu_{E}$ is the electrophoretic mobility and $\sigma_{\mu}$ is its dispersion. Therefore, $\omega_{1 / 2}^{(2)}$ increases linearly with $E$.

Relation (16) explains the peak broadening in the heterodyne autocorrelation function (see Figure 9). In short, for small electric fields, the relaxation time of the correlation functions is dominated by the diffusion coefficient, whereas for high electric fields the frequency dispersion dominates. Another important conclusion is that mobility polydispersity only affects the width of the peaks, whereas the average Doppler frequency does not vary.

On the other hand, due to mobility polydispersity the autocorrelation function decays in a time of order $\sigma_{\omega}^{-1}$. The period of the oscillations of the same function is $\omega_{D}^{-1}$. Both quantities depend on the electric field, as equation (16) shows, but their ratio is independent of it. However, we can 
not measure $\sigma_{\omega}$ directly, but only the value of $\omega_{1 / 2}^{(2)}$. In fact

$$
\frac{1 / \omega_{1 / 2}^{(2)}}{1 / \omega_{D}}=\frac{\mu_{E}}{\sigma_{\mu} \sqrt{2 \ln 2}}
$$

that can be used to calculate the value of $\sigma_{\mu}$.

Although we have assumed a Gaussian distribution of Doppler frequencies, this will not be so in general. However, the main conclusion that the value $\omega_{1 / 2}^{(2)}$ increases with the electric field due to the mobility polydispersity, will remain true. Even more, equation

(17) can be used, at least, as an estimation of the mobility polydispersity $\sigma_{\mu}$.

Table 1: Ratio between the Doppler frequency, $\omega_{D}$, and $\omega_{1 / 2}^{(2)}$. It tends to a constant value close to 0.6. The concentration of particles is $4.5 \%$ in volume.

\begin{tabular}{llll}
\hline$E(\mathrm{KV} / \mathrm{m})$ & $\omega_{D}(\mathrm{rad} / \mathrm{s})$ & $\omega_{1 / 2}^{(2)}(\mathrm{rad} / \mathrm{s})$ & $\frac{\omega_{1 / 2}^{(2)}}{\omega_{D}}$ \\
\hline 25.0 & 320 & 63 & 0.20 \\
27.5 & 352 & 214 & 0.61 \\
30.0 & 364 & 238 & 0.66 \\
32.5 & 434 & 251 & 0.58 \\
35.0 & 439 & 283 & 0.64 \\
\hline
\end{tabular}

Table Table 1 shows how the ratio between the Doppler frequency and $\omega_{1 / 2}^{(2)}$ does not change with the electric field when this is high enough. From the data of table Table 1, we can estimate experimentally the mobility dispersion $\sigma_{\mu}$ if we take $\omega_{1 / 2}^{(2)} / \omega_{D} \approx \sigma_{\mu} / \mu_{E}$. The mobility dispersion results of the order of $0.6 \mu_{E}$.

\section{Discussion}

First, it is worth evaluating the double layer thickness. We did not add electrolyte to the suspension, therefore, the double layer is formed from solvent molecules and impurities. In order to estimate 
the Debye length, we use the expression:

$$
\kappa^{-1}=\sqrt{\frac{D \varepsilon_{r} \varepsilon_{0}}{\sigma}}
$$

where $\varepsilon_{0}$ is the dielectric permittivity of the vacuum, $\varepsilon_{r}$ is the relative permittivity of the tolueneethanol mixture, $D$ is the diffusion coefficient of ions and $\sigma$ the electrical conductivity of the liquid.

Considering a typical value for the diffusion coefficient of order of $10^{-9} \mathrm{~m}^{2} / \mathrm{s}$ and the measured value for the conductivity, we obtain $\kappa^{-1} \approx 90 \mathrm{~nm}$ and $\kappa a \approx 0.44$. Under these conditions particle-particle forces cannot be neglected. In fact, the typical distance between particles varies from $500 \mathrm{~nm}$, for a concentration of $0.36 \%$, to less than $200 \mathrm{~nm}$ for concentrations higher than $4.5 \%$; this leads to overlap of the double layers.

In Shugai's work, ${ }^{4}$ the mobility of suspensions with thick double layer is numerically studied. In addition to $\kappa^{-1}$, another characteristic length enters the pair correlations functions $g(r)$. This is the Bjerrum length

$$
\lambda_{B}=\frac{e^{2}}{4 \pi \varepsilon_{0} \varepsilon_{r} k_{B} T}
$$

In our case, $a \lambda_{B}^{-1} \approx 5$. Shugai $e t a l,{ }^{4}$ for $\kappa a=1$ and $a \lambda_{B}^{-1}=10$, obtained for the parameter of equation (6) $S \approx-6$. The experimental measurement that we have performed is thus in quite good agreement with this numerical result.

Another important point discussed by Ennis ${ }^{9}$ and Shugai ${ }^{4}$ is the following: due to hydrodynamic interactions between pair of particles, the mobility in a direction parallel to the line of their centers is different from the mobility in the transverse direction. As a consequence, in suspension, the particle velocity has fluctuating components, leading to mobility dispersion. For $a \lambda_{B}^{-1}=10$ and $\kappa a=1$, Shungai et al computes the fluctuation in particle mobility $\frac{\sigma_{\mu}}{\mu_{E}} \approx 7 \cdot 10^{-2}$.

For smaller values of $a \lambda_{B}^{-1}$ and $\kappa a$, Shugai et al show that a larger value of $\sigma_{\mu} \mu_{E}^{-1}$ is expected. Therefore, our measurements are in qualitative agreement with the numerical computation.

Other sources of mobility dispersion should be taken in account. First, the $10 \%$ size poly- 
dispersity could have implications in the mobility dispersion. Unfortunately, this has not been numerically explored in the work by Shugai et al. Another factor is the surface charge polydispersity. When $\varphi \rightarrow 0$, the electrophoretic mobility must agree with Henry equation. In our case, where $\kappa a \approx 0.44$, Hückel equation (2) is precise enough to give the zeta potential from the mobility at $\varphi=0$ :

$$
\zeta=(47 \pm 3) \mathrm{mV}
$$

Assuming that the zeta potential is the potential at the particle surface, the total charge over each particle can be estimated from the expression

$$
Q=4 \pi \varepsilon_{0} \varepsilon_{r} a \zeta
$$

We estimate that the number of elementary charges is approximately 10. This small quantity implies that any variation of the electrical charge produces large dispersion of the zeta potential, and, hence, of the mobility.

\section{Conclusion}

In conclusion, the technique of index of refraction matching has allowed us to study the electrophoretic mobility of concentrated suspension by PCS. It has been found that the mobility of the particles decreases with the concentration faster than $1-1.5 \varphi$, which indicates that the interactions between the particles are important. The measured mean mobility is in good agreement with the numerical computing by Shugai et al. This experimental technique enabled us to measure the mobility polydispersity as well. This polydispersity has been seldom measured in the past. We think that this is a useful method to get insight in the velocity fluctuations in electrophoresis. 


\section{Acknowledgement}

We acknowledge fruitful discussions with Dr. Elisabeth Lemaire. We thank André Audoly and Catherine Laye for their technical support. This research has been partially supported by the Spanish Ministerio de Ciencia y Tecnología (MCYT) (FIS2006-03645) and the Junta de Andalucía (FQM-421).

\section{References}

(1) D. C. Henry, Proc. R. Soc. London Ser. A 1931, 133, 106.

(2) R. W. O'Brien and L. R. White, J. Chem. Soc. Faraday Trans. II 1978, 74, 1607.

(3) L. D. Reed and F. A. Morrison, J. Colloid Interface Sci. 1975, 54, 117.

(4) Alexander A. Shugai, Steven L. Carnie, Derek Y. C. Chan and John L. Anderson, J. Colloid Interface Sci. 1997, 191, 357.

(5) J. L. Anderson, J. Colloid Interface Sci. 1981, 82, 248.

(6) C. F. Zukoski IV and D. A. Saville, J. Colloid Interface Sci. 1987, 115, 422.

(7) A. T. Pérez and E. Lemaire, J. Colloid Interface Sci. 2004, 279, 259.

(8) S. Ahualli, A. V. Delgado and C. Grosse, J. Colloid Interface Sci. 2006, 301, 660.

(9) J. Ennis and L. R. White, J. Colloid Interface Sci. 1996, 185, 157.

(10) H. Reiber, T. Köller, T. Palberg, F. Carrique, E. Ruiz-Reina and R. Piazza, J. Colloid Interface Sci. 2007, 309, 315.

(11) R. J. Hunter, Colloids Surfaces A: Physicochem. Eng. Aspects 1998, 141, 37.

(12) J. K. Phalakornkul, A. P. Gast and R. Pecora, Phys. Rev. E 1996, 54, 661. 
(13) W. Stöber and A. Fink, J. Colloid Interface Sci. 1968, 26, 62.

(14) Z. Wu, H. Xiang, T. Kim, M. S. Chun and K. Lee, J. Colloid Interface Sci. 2006, 304, 119.

(15) C. W. Robert Handbook of Chemistry and Physics CRC Press, Inc.: Ohio (1974).

(16) A. P. Philipse and A. Vrij, J. Colloid Interface Sci. 1989, 128, 121.

(17) M. Medrano, A. T. Pérez and C. Soria-Hoyo, J. Phys. D: Appl. 2007, 40, 1477.

(18) M. Medrano, A. T. Pérez and C. Soria-Hoyo, IEEE Transactions on Dielectrics and Electrical Insulation, In press.

(19) Berne B and Pecora R 1976 Dynamic Light Scattering J. Willey ed. (1976).

(20) T. Palberg and H. Versmold, J. Phys Chem. 1989, 93, 5296.

(21) Xu R, Langmuir 1993, 9, 2955.

\section{Appendix}

The autocorrelation function is defined as

$$
C(\tau)=\frac{\langle I(t) I(t+\tau)\rangle}{\langle I(t)\rangle^{2}}
$$

where $I(t)$ is the light intensity at the initial time $t$ and $\tau$ is the time lag. The symbols \langle\rangle denote temporal average.

The correlation function (21) may be expressed in terms of the correlation function of the electric field of the scattered light $g(\tau)$.

For homodyne detection, the correlation function is 19

$$
C_{1}(\tau)=1+\alpha_{1}|g(\tau)|^{2}
$$


and for heterodyne detection

$$
C_{2}(\tau)=1+\alpha_{2} \operatorname{Re}[g(\tau)]
$$

where $\alpha_{1}$ and $\alpha_{2}$ are constants depending on experimental parameters, such as the coherence area, and the ratio between the scattered light and the direct beam intensity, for the heterodyne case. The symbol Re denotes the real part of the complex function $g(\tau)$.

The correlation function of the electric field of the scattered light is

$$
g(\tau)=\frac{\left\langle\vec{E}(t) \cdot \vec{E}^{*}(t+\tau)\right\rangle}{\left\langle\vec{E}(t) \cdot \vec{E}^{*}(t)\right\rangle}
$$

where $\vec{E}$ is the electric field of the light scattered by the scattering volume and $\vec{E}^{*}$ denotes its complex conjugate.

For uncorrelated particles, the equation (24) becomes ${ }^{19}$

$$
g(\vec{q}, \tau)=e^{-\Gamma \tau} e^{i \omega_{D} \tau}
$$

where $\Gamma$ is the product $D q^{2}, \vec{q}$ the scattering vector $\left(\vec{q}=\vec{k}_{i}-\vec{k}_{S}\right), D$ the diffusion coefficient and $\omega_{D}$ is the Doppler frequency.

Using equation (25), the correlation functions (22) and (23) take the form

$$
C_{1}(\tau)=1+\alpha_{1} e^{-2 \Gamma \tau}
$$

and

$$
C_{2}(\tau)=1+\alpha_{2} e^{-\Gamma \tau} \cos \left(\omega_{D} \tau\right)
$$

